\title{
The genome and transcriptome of a freshwater bryozoan Cristatella mucedo
}

\author{
V. Starunov ${ }^{1,2}$, A. Predeus ${ }^{3}$, Y. Barbitov ${ }^{3}$, A. Maltseva ${ }^{1}$, V. Kutyumov ${ }^{1}$, E. Vodiasova ${ }^{4}$, \\ E. Chelebieva ${ }^{4}$, D. Romanova ${ }^{4}$, A. Ostrovsky ${ }^{1}$, L. Moroz ${ }^{4}$ \\ ${ }^{1}$ Saint-Petersburg State University, St. Petersburg, Russia \\ ${ }^{2}$ Zoological Institute of RAS, St. Petersburg, Russia \\ ${ }^{3}$ Bioinformatics institute, St. Petersburg, Russia \\ ${ }^{4}$ Kovalevsky Institute of Marine Biological Research of RAS, Sevastopol, Russia \\ *e-mail:staunov@gmail.com
}

Key words: Bryozoa, Cristatella mucedo, genome, transcriptome

Motivation and Aim: The freshwater bryozoan Cristatella mucedo is a colonial organism from the widespread, but poorly studied phylum Bryozoa. Colonies of bryozoans consist of numerous zooid modules, which possess their own brains, digestive systems, and trapping apparatuses. The phylogenetic position of bryozoans and their relationship with other animal phyla are still rather disputable. Unlike other bryozoans, the colonies of C. mucedo are transparent and do not have mineral skeleton, which makes experimental procedures easier. Moreover, the $C$. mucedo colonies are capable to active directional crawling which is a unique case for colonial invertebrates. Mechanisms of the colony integration are still unknown. The genomic and transcriptomic studies are expected to uncover the molecular basis of integrative mechanisms, uniting individual zooids into a colony. Methods and Algorithms: We performed whole-genome sequencing with long and short reads. Long reads were obtained with Oxford Nanopore Minion instrument; short pairedend reads were obtained with Illumina MiSeq And Hiseq 2500. The assembly was done using Canu and Abruijn software, with subsequent polishing by Nanopolish and Pilon. The RNA sequencing was performed with Illumina HiSeq 2500 instrument and the transcriptomes were assembled with Trinity. The annotation was done by Dammit and OrthoDB, Rfam, and Pfam databases. We also performed single cell RNA sequencing on the young $C$. mucedo colonies using microfluidic device 10-x Genomics for capturing. Results: The genome size of $C$. mucedo is about 600-650 Mb. The hybrid genome assembling with both long and short reads was suggested to be an efficient for eukaryotic genomes and allowed us to build a very high-quality assembly with N50 between 1.1-1.3 Mb. The BUSCO score was more than $90 \%$ from "core metazoa" set. The bulk transcriptome was also of very high quality with BUSCO score more than $97 \%$, and allowed to determine and analyze several important groups of proteins such as neuropeptides, cell adhesion molecules, immune-related molecules, development toolkit genes etc. The single cell sequencing allowed to determine 10 different cellular clusters and find out cluster-specific genes.

Conclusion: Our results make C. mucedo a new emerging model to study different integrative and developmental processes in colonial organisms. This project provides powerful instruments for further molecular, biochemical, neurobiological and evolutionary researches.

Acknowledgements: Scientific research were performed at the Research park of St. Petersburg State University "Biobank", "Center for Molecular and Cell Technologies", "Computing Center", and "Center for Culture Collection of Microorganisms". The research were supported by the Ministry of Education and Science of the Russian Federation grant No. 14.W03.31.0015, and by RSF grant 18-14-00086. 